University of Denver

Digital Commons @ DU

Mathematics Preprint Series

Department of Mathematics

2014

\title{
An Isometric Dynamics for a Causal Set Approach to Discrete Quantum Gravity
}

S. Gudder

Follow this and additional works at: https://digitalcommons.du.edu/math_preprints

Part of the Mathematics Commons

\section{Recommended Citation}

Gudder, S. (2014). An Isometric Dynamics for a Causal Set Approach to Discrete Quantum Gravity. Mathematics Preprint Series. Retrieved from https://digitalcommons.du.edu/math_preprints/10

This Article is brought to you for free and open access by the Department of Mathematics at Digital Commons @ DU. It has been accepted for inclusion in Mathematics Preprint Series by an authorized administrator of Digital Commons@DU. For more information, please contact jennifer.cox@du.edu,dig-commons@du.edu. 


\section{An Isometric Dynamics for a Causal Set Approach to Discrete Quantum Gravity}

\section{Comments}

This is a post-peer-review, pre-copyedit version of an article published in the International Journal of Theoretical Physics. The final authenticated version is available online at: https://doi.org/10.1007/ s10773-014-2398-9 


\title{
AN ISOMETRIC DYNAMICS FOR A CAUSAL SET APPROACH TO DISCRETE QUANTUM GRAVITY
}

\author{
S. Gudder \\ Department of Mathematics \\ University of Denver \\ Denver, Colorado 80208, U.S.A. \\ sgudder@du.edu
}

\begin{abstract}
We consider a covariant causal set approach to discrete quantum gravity. We first review the microscopic picture of this approach. In this picture a universe grows one element at a time and its geometry is determined by a sequence of integers called the shell sequence. We next present the macroscopic picture which is described by a sequential growth process. We introduce a model in which the dynamics is governed by a quantum transition amplitude. The amplitude satisfies a stochastic and unitary condition and the resulting dynamics becomes isometric. We show that the dynamics preserves stochastic states. By "doubling down" on the dynamics we obtain a unitary group representation and a natural energy operator. These unitary operators are employed to define canonical position and momentum operators.
\end{abstract}

\section{Microscopic Picture}

We call a finite poset $(x,<)$ a causet and interpret $a<b$ in $x$ to mean that $b$ is in the causal future of $a$. If $x$ and $y$ are causets with cardinality $|y|=|x|+1$, then $x$ produces $y$ (denoted $x \mapsto y$ ) if $y$ is obtained from $x$ by adjoining a 
single maximal element to $x$. If $x \rightarrow y$ we call $y$ an offspring of $x$. A labeling for a causet $x$ is a bijection

$$
\ell: x \rightarrow\{1,2, \ldots,|x|\}
$$

such that $a, b \in x$ with $a<b$ implies $\ell(a)<\ell(b)$. We then call $x=(x, \ell)$ a labeled causet. A labeling of $x$ corresponds to a "birth order" for the elements of $x$. Two labeled causets $x, y$ are isomorphic if there is a bijection $\phi: x \rightarrow y$ such that $a<b$ in $x$ if and only if $\phi(a)<\phi(b)$ in $y$ and $\ell[\phi(a)]=\ell(a)$ for all $a \in x$. A causet is covariant if it has a unique labeling (up to isomorphisms) and we call a covariant causet a c-causet. Covariance corresponds to the properties of a manifold being independent of the coordinate system used to describe it. Denote the set of $c$-causets with cardinality $n$ by $\mathcal{P}_{n}$ and the set of all $c$-causets by $\mathcal{P}$. It is shown in [3] that any $x \in \mathcal{P}$ with $x \neq \emptyset$ has a unique producer in $\mathcal{P}$ and precisely two offspring in $\mathcal{P}$. It follows that $\left|\mathcal{P}_{n}\right|=2^{n-1}, n=1,2, \ldots$ For more background concerning the causet approach to discrete quantum gravity we refer the reader to $[4,5,7]$. For more information about $c$-causets the reader can refer to $[1,2,3]$.

Two elements $a, b \in x$ are comparable if $a<b$ or $b<a$. We say that $a$ is a parent of $b$ and $b$ is a child of $a$ if $a<b$ and there is no $c \in x$ with $a<c<b$. A path from $a$ to $b$ in $x$ is a sequence $a_{1}=a, a_{2}, \ldots, a_{n-1}, a_{n}=b$ where $a_{i}$ is a parent of $a_{i+1}, i=1, \ldots, n-1$. The height $h(a)$ of $a \in x$ is the cardinality minus one of a longest path in $x$ that ends with $a$. If there is no such path, we set $h(a)=0$. It is shown in [3] that a causet $x$ is covariant if and only if $a, b \in x$ are comparable whenever $h(a) \neq h(b)$.

If $x \in \mathcal{P}$, we call the sets

$$
S_{j}(x)=\{a \in x: h(a)=j\}, j=0,1,2, \ldots
$$

shells and the sequence of integers $s_{j}(x)=\left|S_{j}(x)\right|, j=0,1,2, \ldots$ is the shell sequence for $x$ [1]. A $c$-causet is uniquely determined by its shell sequence and we think of $\left\{s_{j}(x)\right\}$ as describing the "shape" or geometry of $x$. The tree $(\mathcal{P}, \rightarrow)$ can be thought of as a growth model and an $x \in \mathcal{P}_{n}$ is a possible universe at step (time) $n$. An instantaneous universe $x \in \mathcal{P}_{n}$ grows one element at a time in one of two ways. If $x \in \mathcal{P}_{n}$ has shell sequence $\left(s_{0}(x), s_{1}(x), \ldots, s_{m}(x)\right)$, then $x \rightarrow x_{0}$ or $x \rightarrow x_{1}$ where $x_{0}, x_{1}$ have shell sequence $\left(s_{0}(x), s_{1}(x), \ldots, s_{m}(x)+1\right)$ and $\left(s_{0}(x), s_{1}(x), \ldots, s_{m}(x), 1\right)$, respectively. In this way, we recursively order the $c$-causets in $\mathcal{P}$ using the notation 
$x_{n, j}, n=1,2, \ldots, j=0,1,2, \ldots, 2^{n-1}-1$, where $n=\left|x_{n, j}\right|$. For example, in terms of their shell sequences we have:

$$
\begin{aligned}
& x_{1,0}=(1), x_{2,0}=(2), x_{2,1}=(1,1), x_{3,0}=(3), x_{3,1}=(2,1), x_{3,2}=(1,2), x_{3,3}=(1,1,1) \\
& x_{4,0}=(4), x_{4,1}=(3,1), x_{4,2}=(2,2), x_{4,3}=(2,1,1), x_{4,4}=(1,3), x_{4,5}=(1,2,1) \\
& x_{4,6}=(1,1,2), x_{4,7}=(1,1,1,1)
\end{aligned}
$$

In the microscopic picture, we view a $c$-causet as a framework or scaffolding for a possible universe. The vertices of $x$ represent small cells that can be empty or occupied by a particle. The shell sequence for $x$ gives the geometry of the framework. In [1] we have shown how to construct a metric or distance function on $x$. This metric has simple and useful properties. However, the present paper is mainly devoted to the macroscopic picture and the quantum dynamics that can be developed in that picture. Figure 1 illustrates the first four steps of the sequential growth process $(\mathcal{P}, \rightarrow)$. Notice that this is a multiverse model in which infinite paths represent the histories of "completed" universes [4].

\section{Macroscopic Picture}

We now study the macroscopic picture which describes the evolution of a universe as a quantum sequential growth process. In such a process, the probabilities and propensities of competing geometries are determined by quantum amplitudes. These amplitudes provide interferences that are characteristic of quantum systems. A transition amplitude is a map $\widetilde{a}: \mathcal{P} \times \mathcal{P} \rightarrow \mathbb{C}$ satisfying $\widetilde{a}(x, y)=0$ if $x \nrightarrow y$ and $\sum_{y \in \mathcal{P}} \widetilde{a}(x, y)=1$ for every $x \in \mathcal{P}$. Since $x_{n, j}$ only has the offspring $x_{n+1,2 j}$ and $x_{n+1,2 j+1}$ we have that

$$
\sum_{k=0}^{1} \widetilde{a}\left(x_{n, j}, x_{n+1,2 j+k}\right)=1
$$

for all $n=1,2, \ldots, j=0,1,2, \ldots, 2^{n-1}-1$. We call $\widetilde{a}$ a unitary transition amplitude (uta) if $\widetilde{a}$ also satisfies $\sum_{y \in \mathcal{P}}|\widetilde{a}(x, y)|^{2}=1$ or as in (2.1) we have

$$
\sum_{k=0}^{1}\left|\widetilde{a}\left(x_{n, j}, x_{n+1,2 j+k}\right)\right|^{2}=1
$$




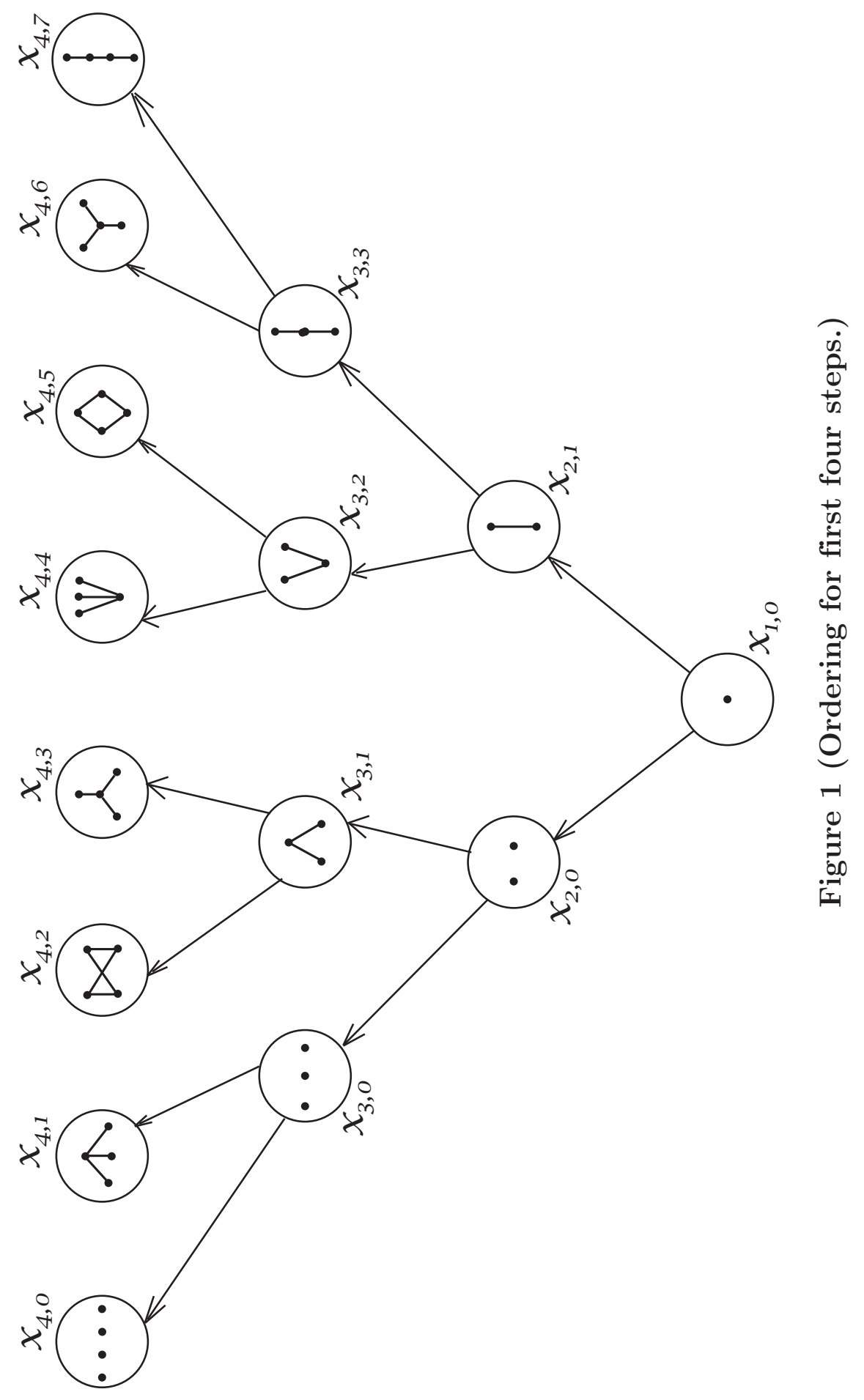


One might suspect that these restrictions on a uta are so strong that the possibilities are very limited. This would be true if $\widetilde{a}$ were real valued. In this case, $\widetilde{a}(x, y)=1$ for one $y$ with $x \rightarrow y$ and $\widetilde{a}(x, y)=0$, otherwise. However, in the complex case, the next result shows that there are a continuum of possibilities.

Theorem 2.1. Two complex numbers $a, b$ satisfy $a+b=|a|^{2}+|b|^{2}=1$ if and only if there exists $a \theta \in[0, \pi)$ such that $a=\cos \theta e^{i \theta}$ and $b=-i \sin \theta e^{i \theta}$. Moreover, $\theta$ is unique.

Proof. Necessity is clear. For sufficiency, suppose the conditions $a+b=$ $|a|^{2}+|b|^{2}=1$ hold. Then

$$
1=|a|^{2}+|b|^{2}=|a|^{2}+|1-a|^{2}=|a|^{2}+(1-a)(1-\bar{a})=1-2 \operatorname{Re} a+2|a|^{2}
$$

Hence, $|a|^{2}=$ Re $a$. Letting $a=|a| e^{i \theta}$ we have that $|a|^{2}=|a| \cos \theta$. If $a=0$, the result holds with $\theta=\pi / 2$. If $a \neq 0$, we have that $|a|=\cos \theta$ and Re $a=|a| \cos \theta$. Hence, $a=\cos \theta e^{i \theta}$ and

$$
\begin{aligned}
b & =1-\cos \theta e^{i \theta}=1-\cos ^{2} \theta-i \cos \theta \sin \theta=\sin \theta(\sin \theta-i \cos \theta) \\
& =-i \sin \theta e^{i \theta}
\end{aligned}
$$

Uniqueness follows from the fact that $\cos \theta$ is injective on $[0, \pi)$.

If $\tilde{a}: \mathcal{P} \times \mathcal{P} \rightarrow \mathbb{C}$ is a uta, we call

$$
c_{n, j}^{k}=\widetilde{a}\left(x_{n, j}, x_{n+1,2 j+k}\right), \quad k=0,1
$$

the coupling constants for $\widetilde{a}$. It follows from Theorem 2.1 that there exist $\theta_{n, j} \in[0, \pi)$ such that

$$
c_{n, j}^{0}=\cos \theta_{n, j} e^{i \theta_{n, j}}, \quad c_{n, j}^{1}=-i \sin \theta_{n, j} e^{i \theta_{n, j}}
$$

It follows that $c_{n, j}^{0}+c_{n, j}^{1}=\left|c_{n, j}^{0}\right|^{2}+\left|c_{n, j}^{1}\right|^{2}=1$ for all $n=1,2, \ldots, j=$ $0,1,2 \ldots, 2^{n-1}-1$. Let $H_{n}$ be the Hilbert space

$$
H_{n}=L_{2}\left(\mathcal{P}_{n}\right)=\left\{f: \mathcal{P}_{n} \rightarrow \mathbb{C}\right\}
$$

with the standard inner product

$$
\langle f, g\rangle=\sum_{x \in \mathcal{P}_{n}} \overline{f(x)} g(x)
$$


A path in $\mathcal{P}$ is a sequence $\omega=\omega_{1} \omega_{2} \cdots$ where $\omega_{i} \in \mathcal{P}_{i}$ and $\omega_{i} \rightarrow \omega_{i+1}$ Similarly, an $n$-path has the form $\omega=\omega_{1} \omega_{2} \cdots \omega_{n}$ where again $\omega_{i} \in \mathcal{P}_{i}$ and $\omega_{i} \rightarrow \omega_{i+1}$. We denote the set of paths by $\Omega$ and the set of $n$-paths by $\Omega_{n}$. Since every $x \in \mathcal{P}_{n}$ has a unique $n$-path terminating at $x$, we can identify $\mathcal{P}_{n}$ with $\Omega_{n}$ and we write $\mathcal{P} \approx \Omega_{n}$. Similarly, we identify $H_{n}$ with $L_{2}\left(\Omega_{n}\right)$. If $\widetilde{a}$ is a uta and $\omega=\omega_{1} \omega_{2} \cdots \omega_{n} \in \Omega_{n}$, we define the amplitude of $\omega$ to be

$$
a(\omega)=\widetilde{a}\left(\omega_{1}, \omega_{2}\right) \widetilde{a}\left(\omega_{2}, \omega_{3}\right) \cdots \widetilde{a}\left(\omega_{n-1}, \omega_{n}\right)
$$

Moreover, we define the amplitude of $x \in \mathcal{P}_{n}$ to be $a(\omega)$ where $\omega \in \Omega_{n}$ terminates at $x$.

Let $\widehat{x}_{n}$, be the unit vector in $H_{n}$ given by the characteristic function $\chi_{x_{n, j}}$. Then clearly, $\left\{\widehat{x}_{n, j}: j=0,1, \ldots, 2^{n-1}-1\right\}$ forms an orthonormal basis for $H_{n}$. Define the operators $U_{n}: H_{n} \rightarrow H_{n+1}$ by

$$
U_{n} \widehat{x}_{n, j}=\sum_{k=0}^{1} c_{n, j}^{k} \widehat{x}_{n+1,2 j+k}
$$

and extend $U_{n}$ to $H_{n}$ by linearity.

Theorem 2.2. (i) The adjoint of $U_{n}$ is given by $U_{n}^{*}: H_{n+1} \rightarrow H_{n}$ where

$$
U_{n}^{*} \widehat{x}_{n+1,2 j+k}=\bar{c}_{n, j}^{k} \widehat{x}_{n, j}, \quad k=0,1
$$

(ii) $U_{n}$ is a partial isometry with $U_{n}^{*} U_{n}=I_{n}$ and

$$
U_{n} U_{n}^{*}=\sum_{j=0}^{2^{n-1}-1}\left|\sum_{k=0}^{1} c_{n, j}^{k} \widehat{x}_{n+1,2 j+k}\right\rangle\left\langle\sum_{k=0}^{1} c_{n, j}^{k} \widehat{x}_{n+1,2 j+k}\right|
$$

Proof. (i) To show that (2.3) holds, we have

$$
\begin{aligned}
\left\langle U_{n}^{*} \widehat{x}_{n+1,2 j^{\prime}+k^{\prime}}, \widehat{x}_{n, j}\right\rangle & =\left\langle\widehat{x}_{n+1,2 j^{\prime}+k^{\prime}}, U_{n} \widehat{x}_{n, j}\right\rangle \\
& =\left\langle\widehat{x}_{n+1,2 j^{\prime}+k^{\prime}}, \sum_{k=0}^{1} c_{n, k}^{k} \widehat{x}_{n+1,2 j+k}\right\rangle \\
& =c_{n, j}^{k} \delta_{j j^{\prime}} \delta_{k k^{\prime}}=\left\langle\bar{c}_{n, j^{\prime}}^{k^{\prime}} \widehat{x}_{n, j^{\prime}} \widehat{x}_{n, j}\right\rangle
\end{aligned}
$$

(ii) To show that $U_{n}^{*} U_{n}=I_{n}$ we have by (i) that

$$
U_{n}^{*} U_{n} \widehat{x}_{n, j}=\sum_{k=0}^{1} c_{n, j}^{k} U_{n}^{*} \widehat{x}_{n+1,2 j+k}=\sum_{k=0}^{1}\left|c_{n, j}^{k}\right|^{2} \widehat{x}_{n, j}=\widehat{x}_{n, j}
$$


Since $\left\{\widehat{x}_{n, j}: j=0,1, \ldots, 2^{n-1}-1\right\}$ forms an orthonormal basis for $H_{n}$, the result follows. Equation (2.4) holds because it is well-known that $U_{n} U_{n}^{*}$ is the projection onto the range of $\mathcal{R}\left(U_{n}\right)$. We can also show this directly as follows

$$
\begin{aligned}
& \sum_{j=0}^{2^{n-1}-1}\left|\sum_{k=0}^{1} c_{n, j}^{k} \widehat{x}_{n+1,2 j+k}\right\rangle\left\langle\sum_{k=0}^{1} c_{n, j^{\prime}}^{k} \widehat{x}_{n+1,2 j+k}\right| \widehat{x}_{n+1,2 j^{\prime}+k^{\prime}} \\
& \quad=\sum_{j=0}^{2^{n-1}-1} \sum_{k=0}^{1} c_{n, j}^{k} \widehat{x}_{n+1,2 j+k} \bar{c}_{n, j^{\prime}}^{k^{\prime}} \delta_{j j^{\prime}}=\bar{c}_{n, j^{\prime}}^{k^{\prime}} \sum_{k=0}^{1} c_{n, j^{\prime}}^{k} x_{n+1,2 j^{\prime}+k} \\
& =\bar{c}_{n, j^{\prime}}^{k^{\prime}} U_{n} \widehat{x}_{n, j^{\prime}}=U_{n} U_{n}^{*} \widehat{x}_{n+1,2 j^{\prime}+k^{\prime}}
\end{aligned}
$$

It follows from Theorem 2.2 that the dynamics $U_{n}: H_{n} \rightarrow H_{n+1}$ for a uta $\widetilde{a}$ is an isometric operator. As usual a state on $H_{n}$ is a positive operator $\rho$ on $H_{n}$ with $\operatorname{tr}(\rho)=1$. A stochastic state on $H_{n}$ is a state $\rho$ that satisfies $\left\langle\rho 1_{n}, 1_{n}\right\rangle=1$ where $1_{n}=\chi_{\mathcal{P}_{n}}$; that is, $1_{n}(x)=1$ for every $x \in \mathcal{P}_{n}$. Notice that $U_{n}^{*} 1_{n+1}=1_{n}$.

Lemma 2.3. (i) If $\rho$ is a state on $H_{n}$, then $U_{n} \rho U_{n}^{*}$ is a state on $H_{n+1}$. (i) If $\rho$ is a stochastic state on $H_{n}$, then $U_{n} \rho U_{n}^{*}$ is a stochastic state on $H_{n+1}$.

Proof. (i) To show that $U_{n} \rho_{n} U_{n}^{*}$ is positive, we have

$$
\left\langle U_{n} \rho U_{n}^{*} \phi, \phi\right\rangle=\left\langle\rho U_{n}^{*} \phi, U_{n}^{*} \phi\right\rangle \geq 0
$$

for all $\phi \in H_{n+1}$. Moreover, by Theorem 2.2(ii) we have

$$
\operatorname{tr}\left(U_{n} \rho U_{n}^{*}\right)=\operatorname{tr}\left(U_{n}^{*} U_{n} \rho\right)=\operatorname{tr}(\rho)=1
$$

(ii) Since $U_{n}^{*} 1_{n+1}=1_{n}$ we have

$$
\left\langle U_{n} \rho U_{n}^{*} 1_{n+1}, 1_{n+1}\right\rangle=\left\langle\rho U_{n}^{*} 1_{n+1}, U_{n}^{*} 1_{n+1}\right\rangle=\left\langle\rho 1_{n} 1_{n}\right\rangle=1
$$

Denoting the time evolution of states by $\rho_{n} \rightarrow \rho_{n+1}$, Lemma 2.3 shows that $\rho \rightarrow U_{n} \rho U_{n}^{*}$ gives a quantum dynamics for states. We now show this explicitly for the transition amplitude. Since

$$
\left\langle\widehat{x}_{n+1,2 j+k}, U_{n} \widehat{x}_{n, j}\right\rangle=c_{n, j}^{k}=\widetilde{a}\left(x_{n, j}, x_{n+1,2 j+k}\right)
$$

we have for every $\omega=\omega_{1} \omega_{2} \cdots \omega_{n} \in \Omega_{n}$ that

$$
a(\omega)=\left\langle\widehat{\omega}_{2}, U_{1} \widehat{\omega}_{1}\right\rangle\left\langle\widehat{\omega}_{3}, U_{2} \widehat{\omega}_{2}\right\rangle \cdots\left\langle\widehat{\omega}_{n}, U_{n-1} \widehat{\omega}_{n-1}\right\rangle
$$

Define the operator $\rho_{n}$ on $H_{n}$ by $\left\langle\widehat{\omega}, \rho_{n} \widehat{\omega}^{\prime}\right\rangle=\overline{a(\omega)} a\left(\omega^{\prime}\right)$ where $\widehat{\omega}=\chi_{\{\omega\}} \in H_{n}$ for any $\omega \in \Omega_{n}$. 
Theorem 2.4. The operator $\rho_{n}$ is a stochastic state on $H_{n}$.

Proof. To show that $\rho_{n}$ is positive we have

$$
\begin{aligned}
\left\langle f, \rho_{n} f\right\rangle & =\left\langle\sum\left\langle\widehat{\gamma}_{i}, f\right\rangle \widehat{\gamma}_{i}, \rho_{n} \sum\left\langle\widehat{\gamma}_{j}, f\right\rangle \widehat{\gamma}_{j}\right\rangle \\
& =\sum \overline{\left\langle\widehat{\gamma}_{i}, f\right\rangle} \sum\left\langle\widehat{\gamma}_{j}, f\right\rangle\left\langle\widehat{\gamma}_{i}, \rho_{n} \widehat{\gamma}_{j}\right\rangle \\
& =\sum \overline{\left\langle\widehat{\gamma}_{i}, f\right\rangle} \sum\left\langle\widehat{\gamma}_{j}, f\right\rangle \overline{a\left(\gamma_{i}\right)} a\left(\gamma_{i}\right) \\
& =\left|\sum a\left(\gamma_{i}\right)\left\langle\widehat{\gamma}_{i}, f\right\rangle\right|^{2} \geq 0
\end{aligned}
$$

To show that $\rho_{n}$ is a state on $H_{n}$ we have that

$$
\begin{aligned}
\operatorname{tr}\left(\rho_{n}\right)= & \sum\left\langle\widehat{\gamma}_{i}, \rho_{n} \widehat{\gamma}_{i}\right\rangle=\sum \overline{a\left(\gamma_{i}\right)} a\left(\gamma_{i}\right)=\sum\left|a\left(\gamma_{i}\right)\right|^{2} \\
= & \sum_{\omega_{2}} \sum_{\omega_{3}} \cdots \sum_{\omega_{n}}\left|\left\langle\widehat{\omega}_{2}, U_{1} \widehat{\omega}_{1}\right\rangle\right|^{2}\left|\left\langle\widehat{\omega}_{3}, U_{2} \widehat{\omega}_{2}\right\rangle\right|^{2} \cdots\left|\left\langle\widehat{\omega}_{n}, U_{n-1} \widehat{\omega}_{n-1}\right\rangle\right|^{2} \\
= & \sum_{\omega_{2}} \sum_{\omega_{3}} \cdots \sum_{\omega_{n-1}}\left|\left\langle\widehat{\omega}_{2}, U_{1} \widehat{\omega}_{1}\right\rangle\right|^{2} \\
& \cdots\left|\left\langle\widehat{\omega}_{n-1}, U_{n-2} \widehat{\omega}_{n-2}\right\rangle\right|^{2} \sum_{\omega_{n}}\left|\left\langle\widehat{\omega}_{n}, U_{n-1} \widehat{\omega}_{n-1}\right\rangle\right|^{2} \\
= & \sum_{\omega_{2}} \sum_{\omega_{3}} \cdots \sum_{\omega_{n-1}}\left|\left\langle\widehat{\omega}_{2}, U_{1} \widehat{\omega}_{1}\right\rangle\right|^{2} \cdots\left|\left\langle\widehat{\omega}_{n-1}, U_{n-2} \widehat{\omega}_{n-2}\right\rangle\right|^{2} \\
& \vdots \\
= & \sum_{\omega_{2}}\left|\left\langle\widehat{\omega}_{2}, U_{1} \widehat{\omega}_{1}\right\rangle\right|^{2}=1
\end{aligned}
$$

Finally, $\rho_{n}$ is stochastic on $H_{n}$ because

$$
\begin{aligned}
\left\langle 1_{n}, \rho_{n} 1_{n}\right\rangle & =\left\langle\sum \widehat{\gamma}_{i}, \rho \sum \widehat{\gamma}_{j}\right\rangle=\sum_{i, j}\left\langle\widehat{\gamma}_{i}, \rho_{n} \widehat{\gamma}_{j}\right\rangle \\
& =\sum_{i, j} \overline{a\left(\gamma_{i}\right)} a\left(\gamma_{j}\right)=\left|\sum a\left(\gamma_{i}\right)\right|^{2}
\end{aligned}
$$


As before, we obtain

$$
\begin{aligned}
\sum_{\omega \in \Omega_{n}} a(\omega)= & \sum_{\omega_{2}} \sum_{\omega_{3}} \cdots \sum_{\omega_{n}}\left\langle\widehat{\omega}_{2}, U_{1} \widehat{\omega}_{1}\right\rangle\left\langle\widehat{\omega}_{3}, U_{2} \widehat{\omega}_{2}\right\rangle \cdots\left\langle\widehat{\omega}_{n}, U_{n-1} \widehat{\omega}_{n-1}\right\rangle \\
= & \sum_{\omega_{2}} \sum_{\omega_{3}} \cdots \sum_{\omega_{n-1}}\left\langle\widehat{\omega}_{2}, U_{1} \widehat{\omega}_{1}\right\rangle\left\langle\widehat{\omega}_{3}, U_{2}, \widehat{\omega}_{2}\right\rangle \cdots\left\langle\widehat{\omega}_{n-1} U_{n-2} \widehat{\omega}_{n-2}\right\rangle \\
& \vdots \\
= & \sum_{\omega_{2}}\left\langle\widehat{\omega}_{2}, U_{1} \widehat{\omega}_{1}\right\rangle=1
\end{aligned}
$$

If $\omega=\omega_{1} \omega_{2} \cdots \omega_{n} \in \Omega_{n}$, we have seen that $\omega_{n}$ produces two offspring $\omega_{n, 0}, \omega_{n, 1} \in \mathcal{P}_{n+1}$. We call the set

$$
(\omega \rightarrow)=\left\{\omega_{1} \omega_{2} \cdots \omega_{n} \omega_{n, 0}, \omega_{1} \omega_{2} \cdots \omega_{n} \omega_{n, 1}\right\} \subseteq \Omega_{n+1}
$$

the one-step causal future of $\omega$. We say that the sequence $\rho_{n}$ is consistent if

$$
\left\langle(\omega \rightarrow)^{\wedge}, \rho_{n+1}\left(\omega^{\prime} \rightarrow\right)^{\wedge}\right\rangle=\left\langle\widehat{\omega}, \rho_{n} \widehat{\omega}^{\prime}\right\rangle
$$

for every $\omega, \omega^{\prime} \in \Omega_{n}$ where $(\omega \rightarrow)^{\wedge}=\chi_{(\omega \rightarrow)}$. Consistency is important because it follows that the probabilities and propensities given by the dynamics $\rho_{n}$ are conserved in time $[2,3]$.

Theorem 2.5. The sequence $\rho_{n}$ is consistent.

Proof. Let $\omega=\omega_{1} \omega_{2} \cdots \omega_{n}, \omega^{\prime}=\omega_{1}^{\prime}, \omega_{2}^{\prime} \cdots \omega_{n}^{\prime} \in \Omega_{n}$ and suppose that $\omega_{n} \rightarrow$ $\omega_{n, 0}, \omega_{n, 1}$ and $\omega_{n}^{\prime} \rightarrow \omega_{n, 0}^{\prime}, \omega_{n, 1}^{\prime}$. We then have

$$
\begin{aligned}
& \left\langle(\omega \rightarrow)^{\wedge}, \rho_{n+1}\left(\omega^{\prime} \rightarrow\right)^{\wedge}\right\rangle \\
& =\left\langle\left(\omega \omega_{n, 0}\right)^{\wedge}+\left(\omega \omega_{n, 1}\right)^{\wedge}, \rho_{n+1}\left[\left(\omega^{\prime} \omega_{n, 0}^{\prime}\right)^{\wedge}+\left(\omega^{\prime} \omega_{n, 1}^{\prime}\right)^{\wedge}\right]\right\rangle \\
& =\left\langle\left(\omega \omega_{n, 0}\right)^{\wedge}, \rho_{n+1}\left(\omega^{\prime} \omega_{n, 0}^{\prime}\right)^{\wedge}\right\rangle+\left\langle\left(\omega \omega_{n, 0}\right)^{\wedge}, \rho_{n+1}\left(\omega^{\prime} \omega_{n, 1}^{\prime}\right)^{\wedge}\right\rangle \\
& +\left\langle\left(\omega \omega_{n, 1}\right)^{\wedge}, \rho_{n+1}\left(\omega^{\prime} \omega_{n, 0}^{\prime}\right)^{\wedge}\right\rangle\left\langle\left(\omega \omega_{n, 1}\right)^{\wedge}, \rho_{n+1}\left(\omega^{\prime} \omega_{n, 1}^{\prime}\right)^{\wedge}\right\rangle \\
& =\overline{a(\omega) \widetilde{a}\left(\omega_{n} \omega_{n, 0}\right)} a\left(\omega^{\prime}\right)\left[\widetilde{a}\left(\omega_{n}^{\prime}, \omega_{n, 0}^{\prime}\right)+\widetilde{a}\left(\omega_{n}^{\prime}, \omega_{n, 1}^{\prime}\right)\right] \\
& +\overline{a(\omega) \widetilde{a}\left(\omega_{n}, \omega_{n, 1}\right)} a\left(\omega^{\prime}\right)\left[\widetilde{a}\left(\omega_{n}^{\prime}, \omega_{n, 0}^{\prime}\right)+\widetilde{a}\left(\omega_{n}^{\prime}, \omega_{n, 1}^{\prime}\right)\right] \\
& =\overline{a(\omega)} a\left(\omega^{\prime}\right)=\left\langle\widehat{\omega}, \rho_{n} \widehat{\omega}^{\prime}\right\rangle
\end{aligned}
$$


The $n$-decoherence functional is the map $D_{n}: 2^{\Omega_{n}} \times 2^{\Omega_{n}} \rightarrow \mathbb{C}$ defined by $[4,5,7]$

$$
D_{n}(A, B)=\left\langle\chi_{B}, \rho_{n} \chi_{A}\right\rangle
$$

The functional $D_{n}(A, B)$ gives a measure of the interference between $A$ and $B$ when the system is in the state $\rho_{n}$. Clearly $D_{n}\left(\Omega_{n}, \Omega_{n}\right)=1, D_{n}(A, B)=$ $\overline{D_{n}(A, B)}$ and $A \mapsto D_{n}(A, B)$ is a complex measure for every $B \in 2^{\Omega_{n}}$. It is also well-known that if $A_{1}, \ldots, A_{n} \in 2^{\Omega_{n}}$, then the matrix with entries $D_{n}\left(A_{j}, A_{k}\right)$ is positive semidefinite [5]. Notice that

$$
D_{n}\left(\{\omega\},\left\{\omega^{\prime}\right\}\right)=\overline{a(\omega)} a\left(\omega^{\prime}\right)
$$

for every $\omega, \omega^{\prime} \in \Omega_{n}$ and

$$
D(A, B)=\sum\left\{\overline{a(\omega)} a\left(\omega^{\prime}\right): \omega \in A, \omega^{\prime} \in B\right\}
$$

Since $\rho_{n}$ is consistent, we have that

$$
D_{n+1}((A \rightarrow),(B \rightarrow))=D_{n}(A, B)
$$

for every $A, B \in 2^{\Omega_{n}}$ where $(A \rightarrow)=\cup\{(\omega \rightarrow): \omega \in A\}$. The corresponding $q$-measure $[2,5,6]$ is the map $\mu_{n}: 2^{\Omega} \rightarrow \mathbb{R}^{+}$defined by

$$
\mu_{n}(A)=D_{n}(A, A)=\left\langle\chi_{A}, \rho_{n} \chi_{A}\right\rangle
$$

It follows that $\mu_{n}\left(\Omega_{n}\right)=1$ and $\mu_{n+1}((A \rightarrow))=\mu_{n}(A)$ for all $A \in 2^{\Omega_{n}}$. Although $\mu_{n}$ is not additive, it satisfies the grade 2-additive condition: if $A, B, C \in 2^{\Omega_{n}}$ are mutually disjoint then $[4,5,6,7]$

$\mu_{n}(A \cup B \cup C)=\mu_{n}(A \cup B)+\mu_{n}(A \cup C)+\mu_{n}(B \cup C)-\mu_{n}(A)-\mu_{n}(B)-\mu_{n}(C)$

Since $\mu_{n}$ is not a measure we do call it a probability but we interpret $\mu_{n}(A)$ as the quantum propensity for the occurrence of $A$. We have discussed in $[2,3]$ ways of extending the $\mu_{n}$ s to a $q$-measure $\mu$ on suitable subsets of $\Omega$.

A uta is completely stationary (cs) with parameter $\theta \in[0, \pi)$ if $\theta_{n, j}=\theta$ for all $n, j$. For example, let $\widetilde{a}$ be cs with parameter 0 . Then the path $x_{1,0} x_{2,0} x_{3,0} \cdots$ has $q$-measure 1 and all other paths have $q$-measure 0 . Now consider a general cs uta $\widetilde{a}$ with parameter $\theta \in(0, \pi)$. When a path "turns left" $\widetilde{a}$ has the value $\cos \theta e^{i \theta}$ and when it "turns right" $\widetilde{a}$ has the value $-i \sin \theta e^{i \theta}$. Hence if $\omega \in \Omega_{n}$ turns left $\ell$ times and right $r$ times we have

$$
a(\omega)=(\cos \theta)^{\ell}(-i)^{r}(\sin \theta)^{r} e^{i n \theta}
$$


We then have

$$
\mu_{n}(\{\omega\})=|a(\omega)|^{2}=|\cos \theta|^{2 \ell}|\sin \theta|^{2 r}
$$

Hence, $\lim _{n \rightarrow \infty} \mu_{n}(\{\omega\})=0$ and is is natural to define $\mu(\{\omega\})=0$.

A vector

$$
v=\sum_{j=0}^{2^{n-1}-1} v_{j} \widehat{x}_{n, j}=\left(v_{o}, v_{1}, \ldots, v_{2^{n-1}-1}\right) \in H_{n}
$$

is called a stochastic state vector if $\|v\|=1$ and $\left\langle v, 1_{n}\right\rangle=1$. we call the vector

$$
\widehat{a}_{n}=\left(a\left(x_{n, o}\right), a\left(x_{n, 1}\right), \ldots, a\left(x_{n, 2^{n-1}-1}\right)\right) \in H_{n}
$$

an amplitude vector. Of course, $\widehat{a}_{n}$ is a stochastic state vector.

Theorem 2.6. (i) If $v \in H_{n}$ is a stochastic state vector, then $U_{n} v \in H_{n+1}$ is also. (ii) $U_{n} \widehat{a}_{n}=\widehat{a}_{n+1}$. (iii) $U_{n}^{*} \widehat{a}_{n+1}=\widehat{a}_{n}$.

Proof. (i) This follows from the fact that $U_{n}$ is isometric and $U_{n}^{*} 1_{n+1}=1_{n}$. (ii) This holds because

$$
\begin{aligned}
U_{n} \widehat{a}_{n} & =U_{n} \sum_{j=0}^{2^{n-1}-1} a\left(x_{n, j}\right) \widehat{x}_{n, j}=\sum_{j=0}^{2^{n-1}-1}\left[a\left(x_{n, j}\right) c_{n, j}^{0} \widehat{x}_{n+1,2 j}+a\left(x_{n, j}\right) c_{n, j}^{1} \widehat{x}_{n+1,2 j+1}\right] \\
& =\sum_{j+0}^{2^{n}-1} a\left(x_{n+1, j}\right) \widehat{x}_{n+1, j}=\widehat{a}_{n+1}
\end{aligned}
$$

(iii) This is obtained from

$$
\begin{aligned}
U_{n}^{*} \widehat{a}_{n+1} & =\sum\left[a\left(x_{n+1,2 j}\right) \widehat{x}_{n+1,2 j}+a\left(x_{n+1,2 j+1}\right) \widehat{x}_{n+1,2 j+1}\right] \\
& =\sum\left[a\left(x_{n+1,2 j}\right) \bar{c}_{n, j}^{0} \widehat{x}_{n, j}+a\left(x_{n+1,2 j+1}\right) \bar{c}_{n, j}^{1} \widehat{x}_{n, j}\right] \\
& =\sum\left[c_{n, j}^{0} a\left(x_{n, j}\right) \bar{c}_{n, j}^{0}+c_{n, j}^{1} a\left(x_{n, j}\right) \bar{c}_{n, j}^{1}\right] \widehat{x}_{n, j} \\
& =\sum a\left(x_{n, j}\right) \widehat{x}_{n, j}=\widehat{a}_{n}
\end{aligned}
$$

Actually, (iii) follows from (ii) in Theorem 2.6 because $\widehat{a}_{n+1}=U_{n} \widehat{a}_{n} \in$ $\mathcal{R}\left(U_{n}\right)$ so $U_{n}^{*} \widehat{a}_{n+1}=U_{n}^{*} U_{n} \widehat{a}_{n}=\widehat{a}_{n}$. Interference in $\mathcal{P}_{n}$ or $\Omega_{n}$ can be described by the nonadditivity of the $q$-measure $\mu_{n}$. We say that $x, y \in \mathcal{P}_{n}$ do not interfere if

$$
\mu_{n}(\{x, y\})=\mu_{n}(\{x\})+\mu_{n}(\{y\})
$$

The next result gives an application of this concept. 
Theorem 2.7. If $x, y \in \mathcal{P}$ have the same producer, then $x$ and $y$ do not interfere.

Proof. Suppose $x=x_{n+1,2 y}, y=x_{n+1,2 j+1}$ so $x, y$ have the same producer $x_{n, j}$. Then

$$
\begin{aligned}
\mu_{n+1}(\{x, y\}) & =|a(x)+a(y)|^{2}=\left|a\left(x_{n, j}\right) c_{n, j}^{0}+a\left(x_{n, j}\right) c_{n, j}^{1}\right|^{2} \\
& =\left|a\left(x_{n, j}\right)\right|^{2}\left|c_{n, j}^{0}+c_{n, j}^{1}\right|^{2}=\left|a\left(x_{n, j}\right)\right|^{2} \\
& =\left|a\left(x_{n, j}\right)\right|^{2}\left[\left|c_{n, j}^{0}\right|^{2}+\left|c_{n, j}^{1}\right|^{2}\right] \\
& =\left|a\left(x_{n, j}\right) c_{n, j}^{0}\right|^{2}+\left|a\left(x_{n, j}\right) c_{n, j}^{1}\right|^{2} \\
& =\left|a\left(x_{n+1,2 j}\right)\right|^{2}+\left|a\left(x_{n+1,2 j+1}\right)\right|^{2}=\mu_{n+1}(\{x\})+\mu_{n+1}(\{y\})
\end{aligned}
$$

Hence, $x$ and $y$ do not interfere.

In general, the noninterference result in Theorem 2.7 does not hold if $x$ and $y$ have different producers. This is shown in the next two examples.

Example 1. For simplicity, suppose the uta is cs so we have just two coupling constants $c^{0}, c^{1}$. We have seen in Theorem 2.7 that $x_{3,0}$ and $x_{3,1}$ do not interfere. In a similar way, we see that $x_{3,0}$ and $x_{3,2}$ do not interfere. We also have that $x_{3,1}$ does not interfere with $x_{3, j}, j=0,1,2$ and $x_{3,2}$ does not interfere with $x_{3, j}, j=0,1,3$. Let us now consider $x_{3,0}$ and $x_{3,3}$. We have that

$$
\begin{aligned}
\mu_{3}\left(\left\{x_{3,0}, x_{3,3}\right\}\right) & =\left|a\left(x_{3,0}\right)+a\left(x_{3,3}\right)\right|^{2}=\left|\left(c^{0}\right)^{2}+\left(c^{1}\right)^{2}\right|^{2} \\
& =\left|\cos ^{2} \theta-\sin ^{2} \theta\right|=\cos ^{2} 2 \theta
\end{aligned}
$$

On the other hand

$$
\begin{aligned}
\mu_{3}\left(\left\{x_{3,0}\right\}\right)+\mu_{2}\left(\left\{x_{3,3}\right\}\right) & =\left|a\left(x_{3,0}\right)\right|^{2}+\mid a\left(\left.x_{3,3}\right|^{2}\right. \\
& =\cos ^{4} \theta+\sin ^{4} \theta=\frac{1}{2}\left(1+\cos ^{2} 2 \theta\right)
\end{aligned}
$$

so $x_{3,0}$ and $x_{3,3}$ interfere, in general.

Example 2. If the uta is not cs, the situation is more complicated and we incur more interference. In the cs case, we saw in Example 1 that $x_{3,0}$ and $x_{3,2}$ do not interfere. However, in this more general case we have

$$
\mu_{3}\left(\left\{x_{3,0}, x_{3,2}\right\}\right)=\left|a\left(x_{3,0}\right)+a\left(x_{3,2}\right)\right|=\left|c_{1,0}^{0} c_{2,0}^{0}+c_{1,0}^{1} c_{2,1}^{0}\right|^{2}
$$


On the other hand

$$
\mu_{3}\left(\left\{x_{3,0}\right\}\right)+\mu_{3}\left(\left\{x_{3,2}\right\}\right)=\left|a\left(x_{3,0}\right)\right|^{2}+\left|a\left(x_{3,2}\right)\right|^{2}=\left|c_{1,0}^{0}\right|^{2}\left|c_{2,0}^{0}\right|^{2}+\left|c_{1,0}^{1}\right|^{2}\left|c_{2,1}^{0}\right|^{2}
$$

But these two quantities do not agree unless

$$
\operatorname{Re}\left(c_{1,0}^{0} c_{2,0}^{0} \bar{c}_{1,0}^{1} \bar{c}_{2,1}^{0}\right)=0
$$

so $x_{3,0}$ and $x_{3,3}$ interfere, in general.

\section{Double-Down To Unitary}

We have seen that corresponding to a uta with coupling constants $c_{n, j}^{k}$ there are isometries $U_{n}: H_{n} \rightarrow H_{n+1}$ that describe the dynamics for a quantum sequential growth process on $(\mathcal{P}, \rightarrow)$. The operators $U_{n}$ cannot be unitary because $H_{n}$ and $H_{n+1}$ are different dimensional Hilbert spaces. However, we can "double-down" the $U_{n}$ to form operators $V_{n+1}: H_{n+1} \rightarrow H_{n+1}$ by

$$
\begin{aligned}
V_{n+1} \widehat{x}_{n+1,2 j} & =c_{n, j}^{0} \widehat{x}_{n+1,2 j}+c_{n, j}^{1} \widehat{x}_{n+1,2 j+1} \\
V_{n+1} \widehat{x}_{n+1,2 j+1} & =c_{n, j}^{1} \widehat{x}_{n+1,2 j}+c_{n, j}^{0} \widehat{x}_{n+1,2 j+1}
\end{aligned}
$$

Theorem 3.1. The operators $V_{n+1}$ are unitary and $V_{n+1} 1_{n+1}=1_{n+1}, n=$ $1,2, \ldots$.

Proof. Since $\left\|V_{n+1} \widehat{x}_{n+1,2 j}\right\|=\left\|V_{n+1} \widehat{x}_{n+1,2 j+1}\right\|=1$ and

$$
\left\langle V_{n+1} \widehat{x}_{n+1,2 j}, V_{n+1} \widehat{x}_{n+1,2 j+1}\right\rangle=\bar{c}_{n, j}^{0} c_{n, j}^{1}+\bar{c}_{n, j}^{1} c_{n, j}^{0}=0
$$

we conclude that $V_{n+1}$ sends an orthonormal basis to an orthonormal basis. Hence, $V_{n+1}$ is unitary. To show that $V_{n+1} 1_{n+1}=1_{n+1}$ we have

$$
\begin{aligned}
V_{n+1} 1_{n+1} & =\sum_{j}\left(V_{n+1} \widehat{x}_{n+1,2 j}+V_{n+1} \widehat{x}_{n+1,2 j+1}\right) \\
& =\sum_{j}\left[\left(c_{n, j}^{0}+c_{n, j}^{1}\right) \widehat{x}_{n+1,2 j}+\left(c_{n, j}^{1}+c_{n, j}^{0}\right) \widehat{x}_{n+1,2 j+1}\right] \\
& =\sum_{j}\left(\widehat{x}_{n+1,2 j}+\widehat{x}_{n+1,2 j+1}\right)=1_{n+1}
\end{aligned}
$$


The unitary operator $V_{2}$ corresponds to the coupling constants $c_{1,0}^{0}, c_{1,0}^{1}$ and relative to the basis $\left\{\widehat{x}_{2,0}, \widehat{x}_{2,1}\right\}$ has the form

$$
V_{2}=\left[\begin{array}{ll}
c_{1,0}^{0} & c_{1,0}^{1} \\
c_{1,0}^{1} & c_{1,0}^{0}
\end{array}\right]
$$

Besides being unitary, $V_{2}$ is doubly stochastic (row and column sums are one). Of course, this is also true of $V_{n}$. By Theorem 2.1, there exists a unique $\theta \in[0, \pi)$ such that $c_{1,0}^{0}=\cos \theta e^{i \theta}, c_{1,0}^{1}=-i \sin \theta e^{i \theta}$. To make $\theta$ explicit, we write $V_{2}=V_{2}(\theta)$.

Lemma 3.2. The operator $V_{2}(\theta)$ has eigenvalues $1, e^{2 i \theta}$ with corresponding unit eigenvectors $2^{-1 / 2}(1,1), 2^{-1 / 2}(1,-1)$.

Proof. By direct verification we have

$$
\begin{aligned}
V_{2}\left[\begin{array}{l}
1 \\
1
\end{array}\right] & =\left[\begin{array}{l}
c_{1,0}^{0}+c_{1,0}^{1} \\
c_{1,0}^{1}+c_{1,0}^{0}
\end{array}\right]=\left[\begin{array}{l}
1 \\
1
\end{array}\right] \\
V_{2}\left[\begin{array}{c}
1 \\
-1
\end{array}\right] & =\left[\begin{array}{l}
c_{1,0}^{0}-c_{1,0}^{1} \\
c_{1,0}^{1}-c_{1,0}^{0}
\end{array}\right]=\left(c_{1,0}^{0}-c_{1,0}^{1}\right)\left[\begin{array}{c}
1 \\
-1
\end{array}\right]
\end{aligned}
$$

But

$$
\begin{aligned}
c_{1,0}^{0}-c_{1,0}^{1} & =c_{1,0}^{0}-\left(1-c_{1,0}^{0}\right)=2 c_{1,0}^{0}-1=2 \cos \theta e^{i \theta}-1 \\
& =2 \cos ^{2} \theta+2 i \cos \theta \sin \theta-1=\cos ^{2} \theta-\sin ^{2} \theta+i \sin 2 \theta \\
& =\cos 2 \theta+i \sin 2 \theta=e^{2 i \theta}
\end{aligned}
$$

We can write the $2^{n}$-dimensional Hilbert space $H_{n+1}$ as

$$
H_{n+1}=H_{2} \oplus H_{2} \oplus \cdots \oplus H_{2}
$$

where there are $2^{n-1}$ summands and the $j$ th summand has the basis $\left\{\widehat{x}_{n+1,2 j}, \widehat{x}_{n+1,2 j+1}\right\}$. In general, $V_{n+1}$ has the form

$$
V_{n+1}\left(\theta_{1}, \theta_{2}, \ldots, \theta_{2^{n-1}}\right)=V_{2}\left(\theta_{1}\right) \oplus V_{2}\left(\theta_{2}\right) \oplus \cdots \oplus V\left(\theta_{2^{n-1}}\right)
$$

It follows from Lemma 3.2 that $V_{n+1}\left(\theta_{1}, \theta_{2}, \ldots, \theta_{2^{n-1}}\right)$ has eigenvalues 1 (with multiplicity $2^{n-1}$ ) and $e^{2 i \theta_{1}}, e^{2 i \theta_{2}}, \ldots, e^{2 i \theta_{2^{n-1}}}$. The unit eigenvectors corresponding to 1 are

$$
2^{-1 / 2}\left(\widehat{x}_{n+1,2 j}+\widehat{x}_{n+1,2 j+1}\right), \quad j=0,1, \ldots, 2^{n-1}-1
$$


and the unit eigenvector corresponding to $e^{2 i \theta j}$ is

$$
2^{-1 / 2}\left(\widehat{x}_{n+1,2 j}-\widehat{x}_{n+1,2 j+1}\right)
$$

Let $\mathcal{S}\left(H_{n+1}\right)$ be the set of operators on $H_{n+1}$ of the form

$$
\mathcal{S}\left(H_{n+1}\right)=\left\{V_{n+1}\left(\theta_{1}, \theta_{2}, \ldots, \theta_{2^{n-1}}\right): \theta_{n} \in[0, \pi)\right\}
$$

Now $[0, \pi)$ forms an abelian group with operations $a \oplus b=a+b(\bmod \pi)$.

Lemma 3.3. For $\theta_{1}, \theta_{2} \in[0, \pi)$ we have $V_{2}\left(\theta_{1}\right) V_{2}\left(\theta_{2}\right)=V_{2}\left(\theta_{1}+\theta_{2}\right)$.

Proof. Since $V_{2}\left(\theta_{1}\right)$ and $V_{2}\left(\theta_{2}\right)$ have the same eigenvectors, they commute and can be simultaneously diagonalized as

$$
V_{2}\left(\theta_{1}\right)=\left[\begin{array}{cc}
1 & 0 \\
0 & e^{2 i \theta_{1}}
\end{array}\right] \quad V_{2}\left(\theta_{2}\right)=\left[\begin{array}{cc}
1 & 0 \\
0 & e^{2 i \theta_{2}}
\end{array}\right]
$$

Hence, if $\theta_{1}+\theta_{2}<\pi$ then

$$
V_{2}\left(\theta_{1}\right) V_{2}\left(\theta_{2}\right)=\left[\begin{array}{cc}
1 & 0 \\
0 & e^{2 i\left(\theta_{1}+\theta_{2}\right)}
\end{array}\right]=V_{2}\left(\theta_{1} \oplus \theta_{2}\right)
$$

and if $\theta_{1}+\theta_{2} \geq \pi$ then $\theta_{1} \oplus \theta_{2}=\theta_{1}+\theta_{2}-\pi$ and we have

$$
V_{2}\left(\theta_{1}\right) V_{2}\left(\theta_{2}\right)=\left[\begin{array}{cc}
1 & 0 \\
0 & e^{2 i\left(\theta_{1}+\theta_{2}-\pi\right)}
\end{array}\right]=V_{2}\left(\theta_{1}+\theta_{2}-\pi\right)=V\left(\theta_{1} \oplus \theta_{2}\right)
$$

We now form the product group $[0, \pi)^{2^{n-1}}=[0, \pi) \times \cdots \times[0, \pi)$ to obtain the following result.

Corollary 3.4. Under operator multiplication, $\mathcal{S}\left(H_{n+1}\right)$ is an abelian group and $\left(\theta_{1}, \ldots, \theta_{2^{n-1}}\right) \mapsto V_{n+1}\left(\theta_{1}, \ldots, \theta_{2^{n-1}}\right)$ is a unitary representation of the group $[0, \pi)^{2^{n-1}}$.

Since $V_{n+1}$ is unitary, there exists a unique self-adjoint operator $K_{n+1}$ on $H_{n+1}$ such that $V_{n+1}=e^{i K_{n+1}}$. We call $K_{n+1}$ a Hamiltonian operator. For $V_{n+1}\left(\theta_{1}, \ldots, \theta_{2^{n-1}}\right.$ the eigenvalues of $K_{n+1}$ are 0 (with multiplicity $2^{n-1}$ ) and $2 \theta_{1}, \ldots, 2 \theta_{2^{n-1}}$. Hence, $\theta_{j}=2^{-1} \lambda_{j}$ where $\lambda_{j}$ is the $j$ th energy value, 
$j=1, \ldots, 2^{n-1}$. This gives a physical significance for the angles $\theta_{j}$. The corresponding eigenvectors are the same as those given for $V_{n+1}$.

It is natural to define the position operator $Q_{n+1}$ on $H_{n+1}$ by $Q_{n+1} f\left(\widehat{x}_{n+1, k}\right)=$ $k$. Thus, $Q_{n+1} \widehat{x}_{n+1,2 j}=2 j$ and $Q_{n+1} \widehat{x}_{n+1,2 j+1}=2 j+1$. Since $Q_{n+1}$ is diagonal, we immediately see that its eigenvalues are $0,1, \ldots, 2^{n}-1$ with corresponding eigenvector $\widehat{x}_{n+1, k}$. It also seems natural to define the canonical momentum operator $P_{n+1}$ on the subspace generated by $\left\{\widehat{x}_{n+1,2 j}, \widehat{x}_{n+1,2 j+1}\right\}$ as

$$
\begin{aligned}
P_{2}\left(\theta_{j}\right) & =V_{2}\left(\theta_{j}\right)^{*} Q_{2}\left(\theta_{j}\right) V_{2}\left(\theta_{j}\right) \\
& =\left[\begin{array}{ll}
\bar{c}_{n, j}^{0} & \bar{c}_{n, j}^{1} \\
\bar{c}_{n, j}^{1} & \bar{c}_{n, j}^{0}
\end{array}\right] \quad\left[\begin{array}{cc}
2 j & 0 \\
0 & 2 j+1
\end{array}\right] \quad\left[\begin{array}{cc}
c_{n, j}^{0} & c_{n, j}^{1} \\
c_{n, j}^{1} & c_{n, j}^{0}
\end{array}\right] \\
& =\left[\begin{array}{cc}
2 j+\left|c_{n, j}^{1}\right|^{2} & c_{n, j}^{0} \bar{c}_{n, j}^{1} \\
\bar{c}_{n, j}^{0} c_{n, j}^{1} & 2 j+\left|c_{n, j}^{0}\right|^{2}
\end{array}\right]=\left[\begin{array}{cc}
2 j+\sin ^{2} \theta_{n, j} & \frac{i}{2} \sin 2 \theta_{n, j} \\
-\frac{i}{2} \sin 2 \theta_{n, j} & 2 j+\cos ^{2} \theta_{n, j}
\end{array}\right]
\end{aligned}
$$

The eigenvalues of $P_{2}\left(\theta_{j}\right)$ are $2 j$ and $2 j+1$ with corresponding unit eigenvectors

$$
\begin{aligned}
& V_{2}\left(\theta_{j}\right)^{*}\left[\begin{array}{l}
1 \\
0
\end{array}\right]=\left[\begin{array}{l}
\bar{c}_{n, j}^{0} \\
\bar{c}_{n, j}^{1}
\end{array}\right] \\
& V_{2}\left(\theta_{j}\right)^{*}\left[\begin{array}{l}
0 \\
1
\end{array}\right]=\left[\begin{array}{l}
\bar{c}_{n, j}^{1} \\
\bar{c}_{n, j}^{0}
\end{array}\right]
\end{aligned}
$$

The complete momentum operator $P_{n+1}$ is given by

$$
P_{n+1}\left(\theta_{1}, \ldots, \theta_{2^{n-1}}\right)=P_{2}\left(\theta_{1}\right) \oplus P_{2}\left(\theta_{2}\right) \oplus \cdots \oplus P_{2}\left(\theta_{2 n-1}\right)
$$

We now compute the commutator

$$
\begin{aligned}
{\left[P_{2}\left(\theta_{j}\right), Q_{2}\left(\theta_{j}\right)\right] } & =P_{2}\left(\theta_{j}\right) Q_{2}\left(\theta_{j}\right)-Q_{2}\left(\theta_{j}\right) P_{2}\left(\theta_{n}\right)=c_{n, j}^{0} \bar{c}_{n, j}^{1}\left[\begin{array}{ll}
0 & 1 \\
1 & 0
\end{array}\right] \\
& =\frac{i}{2} \sin 2 \theta_{j}\left[\begin{array}{ll}
0 & 1 \\
1 & 0
\end{array}\right]
\end{aligned}
$$


The complete commutation relation is

$$
\begin{aligned}
& {\left[P_{n+1}\left(\theta_{1}, \ldots, \theta_{2^{n-1}}\right), Q_{n+1}\left(\theta_{1}, \ldots, \theta_{2^{n-1}}\right)\right]} \\
& \quad=\left[P_{2}\left(\theta_{1}\right), Q_{2}\left(\theta_{1}\right)\right] \oplus \cdots \oplus\left[P_{2}\left(\theta_{2^{n-1}}\right) Q_{2}\left(\theta_{2^{n-1}}\right)\right]
\end{aligned}
$$

As in the Heisenberg uncertainty relation, the number $\left|\left\langle\phi,\left[P_{n+1}, Q_{n+1}\right] \phi\right\rangle\right|$ gives a lower bound for the product of the variances of $P_{n+1}$ and $Q_{n+1}$. We now compute this number for an amplitude state $\widehat{a}_{n+1}$. We have that

$$
\begin{aligned}
\left\langle\widehat{a}_{n+1},\left[P_{n+1}, Q_{n+1}\right] \widehat{a}_{n+1}\right\rangle & \\
& =\sum_{j}\left\langle\left[\begin{array}{c}
a\left(x_{n+1,2 j}\right) \\
a\left(x_{n+1,2 j+1}\right)
\end{array}\right], c_{n, j}^{0} c_{n, j}^{1}\left[\begin{array}{ll}
0 & 1 \\
1 & 0
\end{array}\right]\left[\begin{array}{c}
a\left(x_{n+1,2 j}\right) \\
a\left(x_{n+1,2 j+1}\right)
\end{array}\right]\right\rangle \\
& =\sum_{j} c_{n, j}^{0} \bar{c}_{n, j}^{1}\left[\bar{a}\left(x_{n+1,2 j}\right) a\left(x_{n+1,2 j+1}\right)+\bar{a}\left(x_{n+1,2 j+1}\right) a\left(x_{n+1,2 j}\right)\right] \\
& =\sum_{j} c_{n, j}^{0} \bar{c}_{n, j}^{1}\left|a\left(x_{n, j}\right)\right|^{2}\left[\bar{c}_{n,}^{0} c_{n, j}^{1}+\bar{c}_{n, j}^{1} c_{n, j}^{0}\right]=0
\end{aligned}
$$

This shows that even though $P_{n+1}$ and $Q_{n+1}$ do not commute, there is no lower bound for the product of their variances when the system is in an amplitude state.

\section{References}

[1] S. Gudder, A unified approach for discrete quantum gravity, arXiv: grqc 1403.5338 (2014).

[2] S. Gudder, A covariant causal set approach to discrete quantum gravity, arXiv: gr-qc 1311.3912 (2013).

[3] S. Gudder, The universe as a quantum computer, arXiv: gr-qc 1405.0638 (2014).

[4] J. Henson, Quantum histories and quantum gravity, arXiv: gr-qc 0901.4009 (2009).

[5] R. Sorkin, Quantum mechanics as quantum measure theory, Mod. Phys. Letts. A9 (1994), 3119-3127. 
[6] R. Sorkin, Causal sets: discrete gravity, arXiv: gr-qc 0309009 (2003).

[7] S. Surya, Directions in causal set quantum gravity, arXiv: gr-qc 1103.6272 (2011). 